\title{
OPINIÓN DE ESTUDIANTES DE ENFERMERÍA SOBRE EL EXAMEN CLÍNICO OBJETIVO ESTRUCTURADO
}

\section{NURSING STUDENTS’ OPINION ABOUT THE OBJECTIVE STRUCTURED CLINICAL EXAMINATION}

\author{
Mónica Illesca P.* \\ Mirtha Cabezas G. ${ }^{* *}$ \\ María Teresa Romo P. ${ }^{* * *}$ \\ Patricia Díaz R. ${ }^{* * *}$
}

\begin{abstract}
RESUMEN
Investigación educativa bajo el paradigma cualitativo, a través de un estudio de caso, cuyo objetivo es conocer el significado que tiene para los estudiantes de segundo año de la Carrera de Enfermería el sistema de evaluación ECOE al finalizar la práctica clínica, Módulo Enfermería Básica del Niño, Adolescente y Adulto, del cuarto nivel académico, año 2007. Los estudiantes a partir de sus historias personales individuales, al final del ECOE respondieron una encuesta semiestructurada donde debían mencionar ventajas, desventajas, sugerencias. El análisis de datos siguió el esquema de Miles y Huberman, a través de la reducción progresiva de ellos (separación de unidades, agrupamiento, identificación y clasificación de elementos), disposición, transformación y obtención de conclusiones verificables, validándose a través de la triangulación por investigadores. En las ventajas se identificaron 88 unidades de significado, finalizando en tres dominios cualitativos "proceso de evaluación", "reconocimiento de competencias genéricas adquiridas en el proceso de evaluación ECOE” y "proceso de gestión y administración del ECOE”. En las desventajas hubo 139 unidades de significado, originando dos dominios cualitativos "reconocimiento de competencias genéricas adquiridas en el proceso de evaluación ECOE" y "proceso de gestión y administración del ECOE". Los resultados evidencian que el ECOE al igual que cualquier sistema de evaluación provoca en los estudiantes ansiedad, estrés. Reconocen que mide destrezas, actitudes y favorece la retroalimentación con el examinador. Para futuros ECOE, de acuerdo a las opiniones de los educandos, se debe considerar el momento de su aplicación y minimizar el tiempo de espera para ser examinados.
\end{abstract}

Palabras clave: Educación basada en competencias, competencia clínica, evaluación educacional.

\begin{abstract}
Educational research under qualitative paradigm through a case study aiming to raise awareness of the meaning that OSCE assessment system has for second year Nursing students at the end of the clinical practice of Basic Nursing in child, adolescent and adult modules, given in the fourth level, 2007. At the end of the OSCE and from their own personal stories the students answered a semi-structured survey where they had to mention advantages, disadvantages and suggestions. Data analysis followed the scheme proposed by Miles and Huberman

\footnotetext{
*Enfermera, Oficina de Educación en Ciencias de la Salud (OFECS). Departamento Medicina Interna. Facultad de Medicina. Universidad de La Frontera, Temuco, Chile. Email: millesca@ufro.cl

"* Químico Farmacéutico, Oficina de Educación en Ciencias de la Salud (OFECS). Departamento Ciencias Preclínicas. Facultad de Medicina. Universidad de La Frontera, Temuco, Chile. Email: mcabezas@ufro.cl

${ }^{* * *}$ Enfermera, Departamento Medicina Interna. Facultad de Medicina. Universidad de La Frontera, Temuco, Chile. Email: mtromo@ufro.cl

${ }^{* * * *}$ Enfermera, Departamento Medicina Interna. Facultad de Medicina. Universidad de La Frontera, Temuco, Chile. Email: mdiazr@ufro.cl
} 
(1994), through the progressive reduction of the data (separate units, clustering, identification and classification of items), provision, processing and obtaining verifiable findings, validating them through researcher triangulation. As for the advantages, 88 units of meanings were identified, ending up in three qualitative domains "evaluation process", "recognition of generic skills acquired in the OSCE assessment process" and "administration and process management of the OSCE." 139 units of meanings were identified as disadvantages, creating two qualitative domains: "recognition of generic skills acquired in the OSCE assessment process" and "process management and administration of the OSCE". The results of this study show that the OSCE as well as any system of student assessment results in anxiety and stress. However, they recognize that measures skills, attitudes and encourages feedback from the examiner. For future, according to the student opinions OSCE should consider the time of application and minimize the time out for examination.

Key words: Competency-based education, clinical competence, educational measurement.

Fecha recepción: 23/11/10 Fecha aceptación: 18/04/11

\section{INTRODUCCIÓN}

Como resultado del desarrollo sin precedente en la historia de la humanidad, de los avances científico-tecnológico, tecnología de la información y la internacionalización, las instituciones de Educación Superior se han visto enfrentadas a responder a los desafíos de una transición que va de la sociedad "de la información" a una denominada "del conocimiento" (1). Para potenciar la capacidad de producción de nuevos saberes, habilidad para adaptar y adoptar nuevas formas didácticas, se debe cambiar el tradicional rol docente así como el del estudiante.

Para ello, el profesorado ha debido desaprender lo aprendido y producto de una profunda reflexión ha buscado nuevas estrategias didácticas para su quehacer educativo con el propósito de lograr en los educandos un aprendizaje significativo, con oportuna retroalimentación acorde a sus esfuerzos, autónomos, aprendedores activos y de por vida para solucionar problemas complejos, con capacidad de trabajo en equipo. En síntesis las competencias tanto genéricas como específicas (2) que les demanda el mundo laboral con nuevos enfoques holísticos del cuidado.

El constructivismo y la andragogía (3) constituyen las bases para sustentar el cambio en la didáctica "centrada en el estudian- te", significando que asuman la responsabilidad de su propio aprendizaje con participación activa en el proceso, de acuerdo a sus propios ritmos y estilo, sumando sus necesidades educacionales a lo formulado por los expertos, quienes han definido los contenidos de formación.

Desde el punto de vista de los hallazgos de la neurociencia, la actividad cerebral se relaciona con la conducta y el aprendizaje en el ser humano involucrando, por lo tanto, al proceso educativo (4).

Todo este nuevo paradigma lleva implícito considerar nuevas formas de evaluación donde se incluye el proceso y el logro de resultados (5). La primera, llamada también formativa, se hace durante todo el transcurso del aprendizaje y su fin es mejorar el mismo, facilitando la adquisición de conocimientos y actitudes (6). En otras palabras, permite valorar a los estudiantes en relación al progreso personal incluyendo comunicación, colaboración y participación, así como también al docente, la asignatura y los recursos utilizados. De este modo, se tiene información tanto de lo positivo como de las dificultades para que en último término la toma de decisiones se realice en forma oportuna para adaptar las estrategias didácticas (7).

El logro de resultados o sumativa, se hace al final del aprendizaje y su fin es acreditar requisitos de titulación o de capacitación 
profesional, mide el nivel de formación alcanzado y si éste es considerado como óptimo (6).

La experiencia docente permite sugerir que los planificadores consideren la correlación entre las estrategias didácticas y el sistema de evaluación. Para este último aspecto es importante utilizar diferentes técnicas con los instrumentos más representativos de ellas, para que su aplicación resulte pertinente y de este modo se propicien las condiciones que permitan obtener la información cualitativa y cuantitativa necesaria. La técnica es el procedimiento mediante el cual se lleva a cabo la apreciación del aprendizaje y el instrumento es el medio con el que el docente obtiene la información requerida (8).

Indudablemente, que la correcta conceptualización de lo anterior incidirá en el compromiso que se adquiere frente al aseguramiento de la calidad de la institución (9).

No menos cierto, el factor estrés que se genera en los estudiantes frente a una evaluación está siempre presente. En general éste se produce toda vez que existe una interacción dinámica entre la valoración cognoscitiva que cada persona hace de las demandas ambientales (exámenes) y las estrategias de afrontamiento para responder a dichas demandas (10).

La evaluación de las competencias disciplinares y genéricas de las/os estudiantes de Enfermería durante las experiencias clínicas es gradual, es decir, recibe retroalimentación diaria de su quehacer con usuarios asignados durante un periodo determinado, para finalmente ser calificado. Esto ha sido por mucho tiempo un desafío, para lo cual es preciso hacer énfasis en la definición de lo que se estima deberían lograr los educandos, así como en las técnicas e instrumentos para evaluarlos, tradicionalmente los utilizados no siempre cumplen con los criterios de objetividad, validez y confiabilidad (11), proporcionando información errónea para la toma de decisiones.

Sin embargo, durante las últimas tres dé- cadas han surgido instrumentos que cumplen con propiedades psicométricas, entre los cuales se encuentra el Examen Clínico Objetivo Estructurado (Objetive Structured Clinical Examination, OSCE) $(12,13)$, el que ha sido aceptado internacionalmente y por ello validado para los cursos de pregrado (14-18) como en los de postítulo y/o postgrado (19-22).

Se utiliza para evaluar el razonamiento clínico (23) en forma planificada, componentes de la competencia profesional incluyendo conocimientos, habilidades clínicas y actitudes. Se aplica a un gran número de educandos durante un mismo tiempo, dando como resultado mayor objetividad al examen. Se estructuran 10 a 20 estaciones, que conforman un circuito donde los estudiantes se exponen en forma rotativa y simultánea a una multiplicidad de tópicos a evaluar sobre la base de los objetivos de aprendizaje de la asignatura. En esta secuencia ellos disponen de cinco minutos como máximo para responder cada aspecto solicitado $(24,25)$.

La evaluación es definida a través de pautas o lista de chequeo estandarizado, especificando el sistema de puntaje (26). La confiabilidad de éstos se basa en que un mismo evaluador utiliza un set estándar de criterios para calificar a los estudiantes, disminuyendo la subjetividad $(12,27)$.

La Carrera de Enfermería de la Universidad de La Frontera, Temuco-Chile, considerando las teorías, paradigmas antes mencionados y las tendencias educacionales para la formación de los profesionales del área de la salud, desde el año 2003 innovó su plan de estudio, de forma tal que aplica un modelo centrado en el estudiante, estructurado en módulos integrados por diversas disciplinas, con aprendizaje basado en problemas en sesiones tutoriales, clases expositivas, talleres y prácticas clínicas, desde el inicio de la formación. Todo ello con instrumentos de evaluación concordante al modelo pedagógico. Es así como para el proceso se aplican pautas de apreciación tanto para la autoeva- 
luación, co-evaluación y evaluación al tutor. Para el resultado del aspecto teórico: ensayo modificado, salto triple, informes. En la práctica clínica, para el proceso se aplica la evaluación formativa semanal con la participación de los actores implicados y para el resultado escalas de apreciación, listas de cotejo, demostración de procedimientos, casos clínicos y ECOE.

El nuevo modelo educativo de la carrera centrado en el estudiante, el ambiente de aprendizaje clínico complejo y estresante, y las ventajas del ECOE para certificar al final de la práctica clínica las competencias adquiridas en los cuatro dominios del saber, motivó la incorporación de esta técnica evaluativa el año 2007 para una actividad curricular profesional integrada.

Con el objetivo de comprender en profundidad el significado que tuvo para los estudiantes ser evaluados a través del ECOE al finalizar la práctica clínica, se formularon los siguientes objetivos orientadores:

- Develar las ventajas y desventajas del ECOE en los cuatro dominios del saber.

- Indagar el aspecto administrativo del examen.

- Identificar posibles sugerencias.

En la literatura se destacan resultados satisfactorios de la utilización del ECOE para evaluar competencias profesionales en las enfermeras/os. Sin embargo, al igual que los exámenes tradicionales produce cierto nivel de estrés en los estudiantes $(28,29)$.

\section{MATERIAL Y MÉTODO}

Investigación educativa desde el paradigma cualitativo a través de un estudio de caso, respaldado por la perspectiva hermenéutica.

En lo concreto, para la presente investigación, el estudio de casos permite elaborar una visión holística y detallada del objeto de estudio (30), el cual se orienta a conocer el significado que tiene para los estudiantes de segundo año de la Carrera de Enfermería el sistema de evaluación ECOE.

El caso es la evaluación al final de la práctica clínica, mediante el ECOE en el Módulo Integrado "Enfermería Básica del Niño, Adolescente y Adulto" impartido en el segundo año del 2007, de carácter teórico-práctico (4/7 h), obligatoria, con una duración de 16 semanas, donde los estudiantes realizan prácticas clínicas en las áreas de Pediatría (20 h), Cirugía-Esterilización (20 h) Medicina $(80 \mathrm{~h})(31)$.

El propósito del Módulo es la Gestión del Cuidado de Enfermería a nivel básico, intrahospitalario, aplicando el Proceso Enfermero en Niños, Adolescentes y Adultos en forma holística, haciendo hincapié en los principios de bioética y reconociendo tanto al equipo de salud como el de enfermería (31).

La organización del ECOE estuvo a cargo de dos docentes. Se estructuraron 13 estaciones: 8 para demostración de habilidades clínicas y actitudinales (vía venosa con tapón; curación simple; preparación solución parenteral; administración subcutánea de fármacos; confección de cama en dos tiempos; control de temperatura, control de respiración y pulso; postura de guantes) y 5 orientadas al dominio cognoscitivo (preparación preoperatorio en general; división topográfica del abdomen; referencias anatómicas de la administración de fármaco intramuscular; administración de medicamentos y administración de oxígeno para usuario pediátrico). Cada una con su respectiva pauta de evaluación, las que fueron elaboradas con el consenso del equipo examinador, compuesto por 10 personas: 7 evaluadores para las estaciones de demostración de habilidades clínicas y actitudinales, una paciente simulada para la estación de control de signos vitales estaciones, una persona para cronometrar el tiempo y una para acompañar a los estudiantes en sala antes del examen. Cabe mencionar 
que las docentes organizadoras del examen formaron parte del equipo evaluador.

Se habilitaron 3 salas para las 13 estaciones, de las cuales cinco estaban orientadas a evaluar el dominio cognoscitivo, sin evaluador, ya que el estudiante dejaba la respuesta en buzones.

Los sujetos de estudio fueron alumnos de la Carrera de Enfermería, inscritos en la actividad curricular 2007, quienes a partir de sus historias personales individuales y colectivas proporcionaron información válida, relacionadas a los objetivos de la investigación.

La muestra, fue por conveniencia de casos por criterios, conformada por todos los estudiantes -53- de segundo año con la condición de haber finalizado la práctica clínica del Módulo Enfermería Básica del Niño, Adolescente y Adulto.

La recolección de datos se obtuvo mediante una encuesta semiestructurada, con preguntas abiertas orientadas a develar por parte de los estudiantes las ventajas, desventajas y sugerencias, la cual fue respondida al final del ECOE y aplicada por los investigadores. Las preguntas orientadoras se referían a: de acuerdo a su experiencia vivida en esta evaluación ¿puede mencionar ventajas?, ¿desventajas?; ¿puede emitir sugerencias para mejorar este examen?

El análisis de datos fue el de comparaciones constantes de Glaser y Strauss (32), no agrupándose en categorías predeterminadas, sino más bien emergiendo de un proceso de razonamiento inductivo. Corresponde a un método generativo, constructivo e inductivo en el que se combina la codificación inductiva de categorías con la comparación constante de ellas. Se adoptó el esquema propuesto por Miles y Huberman (33), a través de la reducción progresiva de la información concordante con las interrogantes del estudio (separación de unidades, agrupamiento, identificación y clasificación de elementos), disposición, transformación y obtención de conclusiones verificables, utilizando metodología propuesta por Van Maanen (34):
- Nivel 1: Identificación y segmentación de narraciones textuales para agrupar en categorías descriptivas (primera aproximación a núcleos temáticos o emergentes).

- Nivel 2: Dominios cualitativos a través de la identificación de similitudes estructurales y elementos comunes de las categorías del Nivel 1.

- Nivel 3: Identificación de dominios cualitativos (análisis secuencial y transversal de las metacategorías).

La rigurosidad científica se garantizó utilizando criterios de rigor Guba y Lincoln (35): credibilidad (triangulación por los cuatro investigadores), dependencia (réplica paso a paso, permitiendo juzgar el grado de correspondencia con otros contextos), confirmabilidad (ejercicio de reflexión y consenso con otros investigadores) y transferibilidad (recogida abundante información descriptiva y desarrollo de descripciones minuciosas).

El aspecto ético en esta investigación educativa se avala en el Consentimiento Informado de los estudiantes y autorización explícita de la directora de la Carrera de Enfermería (36).

\section{RESULTADOS}

Nivel 1, en las ventajas (Tabla 1) se identificaron 88 unidades de significado relevantes, agrupadas en 4 categorías descriptivas emergentes: "instrumentos de evaluación", "administración", "contenidos evaluados" y "actitud del estudiante". A su vez, en las desventajas (Tabla 2) se manifiestan 139 unidades de significado, constituyéndose 6 categorías descriptivas: "actitud del estudiante", "administración", "tiempo", "fecha", "contenidos evaluados" y "relación profesor-alumno".

Nivel 2, representa los núcleos temáticos emergentes o metacategorías que surgen del nivel 1 (considerando aquéllas con una fre- 
Tabla 1. Distribucion de frecuencias de las unidades de significado en relación a las ventajas.

\begin{tabular}{|c|c|l|c|}
\hline No & CÓDIGO & \multicolumn{1}{|c|}{ CATEGORÍA } & $\begin{array}{c}\text { FRECUENCIA UNIDADES } \\
\text { DE SIGNIFICADO }\end{array}$ \\
\hline 1 & EVA & Instrumentos de evaluación & 33 \\
\hline 2 & ADM & Administración & 23 \\
\hline 3 & CONE & Contenidos evaluados & 18 \\
\hline 4 & ACE & Actitud del estudiante & 14 \\
\hline & Total & 88 \\
\cline { 2 - 4 }
\end{tabular}

Tabla 2. Distribucion de frecuencias de las unidades de significado en relacion a las desventajas.

\begin{tabular}{|c|c|l|c|}
\hline No & CÓDIGO & \multicolumn{1}{|c|}{ CATEGORÍA } & $\begin{array}{c}\text { FRECUENCIA UNIDADES } \\
\text { DE SIGNIFICADO }\end{array}$ \\
\hline 1 & ACE & Actitud del estudiante & 65 \\
\hline 2 & ADM & Administración & 31 \\
\hline 3 & TIE & Tiempo & 22 \\
\hline 4 & FEC & Fecha & 10 \\
\hline 5 & CONE & Contenidos evaluados & 8 \\
\hline 6 & RPA & Relación profesor alumno & 3 \\
\hline & Total & & 139 \\
\cline { 2 - 4 }
\end{tabular}

cuencia mayor a 15 unidades de significado). En las ventajas tres metacategorías: "relación entre los contenidos evaluados e instrumento utilizado", "competencias genéricas" y "organización de la actividad” (Tabla 3). En las desventajas dos: "organización de la actividad" y "competencias genéricas" (Tabla 4).
Nivel 3, posterior al análisis secuencial y transversal de las metacategorías, emergen los dominios cualitativos para las ventajas y desventajas. Los núcleos temáticos o centros de interés de los estudiantes de la Carrera de Enfermería con respecto a las ventajas de ser evaluados a través del ECOE permiten dar el

Tabla 3. Relación entre metacategorías, categorías y unidades de significado con respecto a las ventajas.

\begin{tabular}{|l|l|c|c|}
\hline \multirow{2}{*}{ METACATEGORÍAS } & \multicolumn{1}{c|}{ CATEGORÍAS } & \multicolumn{2}{c|}{ UNIDADES DE SIGNIFICADO } \\
\cline { 3 - 4 } & & \multicolumn{2}{c|}{ No } \\
\hline \multirow{2}{*}{$\begin{array}{l}\text { Relación entre los contenidos evaluados e e } \\
\text { instrumento utilizado }\end{array}$} & Contenidos evaluados & 18 & 20.5 \\
\cline { 2 - 4 } & Instrumentos de evaluación & 33 & 37.5 \\
\hline Competencias genéricas & Actitud del estudiante & 14 & 15.9 \\
\hline Organización de la actividad & Administración & 23 & 26.1 \\
\hline Total & & 88 & 100 \\
\hline
\end{tabular}


Tabla 4. Relación entre metacategorías, categorías y unidades de significado con respecto a las desventajas.

\begin{tabular}{|l|l|c|c|}
\hline \multirow{1}{*}{ METACATEGORÍAS } & \multicolumn{1}{|c|}{ CATEGORÍAS } & \multicolumn{2}{c|}{ UNIDADES DE SIGNIFICADO } \\
\cline { 3 - 4 } & & $\mathbf{N o}$ & $\%$ \\
\hline \multirow{3}{*}{ Organización de la actividad } & Fecha & 22 & 7,8 \\
\cline { 2 - 4 } & Tiempo & 31 & 17,2 \\
\cline { 2 - 4 } & Administración & 65 & 24,2 \\
\hline Competencias genéricas & Actitud del estudiante & 128 & 50,8 \\
\hline \multirow{2}{*}{ Total } & & & 100 \\
\hline
\end{tabular}

primer paso de reordenamiento de categorías, dando lugar a los siguientes dominios cualitativos: "Proceso de evaluación", "Proceso de gestión y administración del ECOE” y "Reconocimiento de competencias genéricas adquiridas en el proceso de evaluación ECOE” (Tabla 5).

Con respecto a las desventajas, surgen dos dominios cualitativos: "Proceso de gestión y administración del ECOE" y "Reconocimiento de competencias genéricas adquiridas en el proceso de evaluación ECOE" (Tabla 6).

Para futuros ECOE, de acuerdo a las sugerencias de los educandos se debe considerar el momento de su aplicación (realizarlo al término de la práctica clínica) y conocer previamente las pautas de los procedimientos que se van a evaluar.

Tabla 5. Dominios cualitativos para las ventajas.

\begin{tabular}{|c|c|c|c|c|}
\hline \multirow[t]{2}{*}{ DOMINIOS } & \multirow[t]{2}{*}{ METACATEGORÍAS } & \multirow[t]{2}{*}{ CATEGORÍAS } & \multicolumn{2}{|c|}{ UNIDADES DE SIGNIFICADO } \\
\hline & & & No & $\%$ \\
\hline \multirow{2}{*}{$\begin{array}{l}\text { Dominio 1. "Pro- } \\
\text { ceso de evalua- } \\
\text { ción" }\end{array}$} & \multirow{2}{*}{$\begin{array}{l}\text { Relación entre los } \\
\text { contenidos evaluados } \\
\text { e instrumento } \\
\text { utilizado }\end{array}$} & Contenidos evaluados & 18 & 20,5 \\
\hline & & Instrumentos de evaluación & 33 & 37,5 \\
\hline $\begin{array}{l}\text { Dominio 2. "Pro- } \\
\text { ceso de gestión y } \\
\text { administración } \\
\text { del ECOE" }\end{array}$ & $\begin{array}{l}\text { Organización de la } \\
\text { actividad }\end{array}$ & Administración & 23 & 26,1 \\
\hline $\begin{array}{l}\text { Dominio 3. "Re- } \\
\text { conocimiento de } \\
\text { competencias ge- } \\
\text { néricas adquiridas } \\
\text { en el proceso de } \\
\text { evaluación ECOE" }\end{array}$ & $\begin{array}{l}\text { Competencias } \\
\text { genéricas }\end{array}$ & Actitud del estudiante & 14 & 15,9 \\
\hline \multicolumn{3}{|l|}{ Total } & 88 & 100 \\
\hline
\end{tabular}


Tabla 6. Dominios cualitativos para las desventajas.

\begin{tabular}{|l|l|l|c|c|}
\hline \multicolumn{1}{|c|}{ DOMINIOS } & \multicolumn{1}{c|}{ METACATEGORÍAS } & CATEGORÍAS & \multicolumn{2}{c|}{ UNIDADES DE SIGNIFICADO } \\
\cline { 3 - 5 } & & & No & $\%$ \\
\hline $\begin{array}{l}\text { Dominio 2. "Pro- } \\
\text { ceso de gestión y } \\
\text { administración del } \\
\text { ECOE" }\end{array}$ & $\begin{array}{l}\text { Organización de la acti- } \\
\text { vidad }\end{array}$ & Administración & 31 & 24,2 \\
\cline { 3 - 5 } & Tiempo & 22 & 17,2 \\
\cline { 3 - 5 } & Fecha & 10 & 7,8 \\
\hline $\begin{array}{l}\text { Dominio 3. "Re- } \\
\text { conocimiento de } \\
\text { competencias gené- } \\
\text { ricas adquiridas en } \\
\text { el proceso de eva- } \\
\text { luación ECOE" }\end{array}$ & Competencias genéricas & $\begin{array}{l}\text { Actitud del estu- } \\
\text { diante }\end{array}$ & 65 & \\
\hline & & & & \\
\hline Total & & & 100 \\
\hline
\end{tabular}

En cuanto al aspecto administrativo, sugieren una mejor organización para minimizar el tiempo de espera para ser examinados y cinco de los 53 plantean eliminarlo.

\section{DISCUSIÓN Y COMENTARIO}

En este estudio, los factores considerados por ellos en el dominio cualitativo "Reconocimiento de competencias genéricas" fueron de diferente índole: como desventaja, mencionan el tiempo de espera para ser examinados, el tiempo establecido en cada una de las estaciones para demostrar competencias y el momento del semestre para su realización, el cual se planificó una vez que finalizaron todos los estudiantes su práctica clínica. De todo lo mencionado se puede inferir desconocimiento general por parte de los estudiantes del proceso implícito en un ECOE. En relación al tiempo de espera, esto no pudo ser menor por la cantidad de estudiantes del curso, lo que se podría revertir si se duplicaran las estaciones. Situación no factible por déficit de espacio físico, insumos y recurso humano. En relación al tiempo en cada estación, se consideró lo establecido en la correspondiente literatura $(24,25)$.

Como ventaja en el dominio cualitativo "Reconocimiento de competencias genéricas" mencionan que permite el "desarrollo de habilidad mental", "sistematizar procesos", "identificar debilidades y fortalezas", "mejorar errores". Es destacable consignar que los estudiantes hayan desarrollado estas capacidades, por las cuales apostó la Carrera de Enfermería en la innovación curricular, implementada en el año 2003. Cabe mencionar que en paralelo se publica los postulados del Proyecto Tuning (2).

Es valorable que los estudiantes, en relación al dominio cualitativo "Proceso de evaluación”, destaquen positivamente la calidad de la retroalimentación recibida posteriormente dada por el evaluador (26), hecho que si bien es cierto se produce en las experiencias clínicas, aquí en cinco minutos igual se realiza, demostrando el expertizaje al docente. Otro comentario apunta a que "la misma docente evalúa una estación” ratificando la objetividad del examen $(24,25)$.

A pesar de las adversidades percibidas 
por los estudiantes en el dominio cualitativo "Proceso de gestión y administración", reconocen pertinencia y coherencia del examen respecto a los objetivos formulados en el programa, buena organización del mismo, entretenido y dinámico (37). Además, reconocen que mide destrezas, actitudes y favorece la retroalimentación con el examinador $(24,25)$.

Analizando las sugerencias de los estudiantes, aparte del desconocimiento de este tipo de evaluación a futuro, los docentes deben considerar un tiempo previo de formación para que se enfrenten al examen. En relación a que se realice al término de la práctica clínica de cada grupo, como ellos lo comentan, es imposible por la cantidad de estudiantes y el sistema de rotación (grupos de 5 alumnos durante cuatros semanas). Por supuesto que siendo una forma de examinar nueva en la carrera siempre habrá estudiantes que se resistan a ello.

En consecuencia, este examen refuerza el modelo curricular actual de la carrera, cobrando sentido las teorías de la neurociencia y el constructivismo como el modelo andragógico, lo que se expresa en el significado que tiene para los estudiantes en su proceso enseñanza-aprendizaje, hecho no visualizado en el modelo tradicional empleado anteriormente, sobre todo en lo que es su autoevaluación y retroalimentación por parte del docente (24).

\section{REFERENCIAS}

1. Tünnermann C. La universidad latinoamericana ante los retos del siglo XXI. México: Colecciones UDUAL [Internet]; 2003 [citado 4 de julio 2010]. Disponible en http://www.udual.org/CIDU/ColUDUAL/Tunner/IndicePrologo.pdf .

2. González J, Wagenaar R. Tuning Educational Structures in Europe. Informe Final. Proyecto Piloto-Fase 1. España: Só- crates; 2003.

3. Knowles M. Andragogy in Action. San Francisco: Jossey-Bass; 1984.

4. Salas R. ¿La educación necesita realmente de la neurociencia? Estud pedagóg. 2003; (29): 155-171.

5. Carretero M. Constructivismo y Educación. 8va ed. Buenos Aires: Aique; 2001.

6. Tutosaus J. Sistemas de evaluación de los residentes. Rol del tutor en los mismos. Educ méd [Internet] 2003 [citado 21 octubre 2010]; 6 (3). Disponible en http:// scielo.isciii.es

7. Martínez JM. Los métodos de evaluación de la competencia profesional: la evaluación clínica objetiva estructurada (ECOE). Educ méd. 2005; 8(suppl 2): 18-22.

8. Técnicas e instrumentos para realizar la evaluación del aprendizaje [Internet]; 2008 [citado 9 agosto 2010]. Disponible en: http://www.scribd.com

9. Kemmis S, Stake RE. Evaluating Curriculum. Victoria: Deakin University Press; 1988.

10. Lazarus R, Folkamn S. Estrés y procesos cognitivos. Barcelona: Martínez Roca; 1986.

11. Sloan D, Donelly M, Schwartz R, Stordel W. The Objetive Structured Clinical Examination. The New Gold Standard for Evaluating Postgraduate Clinical Perfomance. Ann Surg. 1995; 222(6): 735-42.

12. Harden RM, Gleeson FA. Assessment of clinical competence using an objetive structured clinical examination. Med Educ. 1979; 13(1): 41-54.

13. Carraccio C, Englander R. The Objetive Structured Clinical Examination. A Step in the Direction of Competency-Based Evaluation. Arch Pediatr Adolesc Med. 2000; 154(7): 736-41.

14. Mavis BE, Cole BL, Hoppe RB. A Survey of Information Sources Used for Progress Decisions about Medical Students. Med Educ Online [Internet] 2000 [citado 9 agosto 2010]; 5(10). Disponible en http://www.med-ed-online.org 
15. Townsend AH, Mcllvenny S, Miller CJ, Dunn EV. The use of an objetive structured clinical examination (OSCE) for formative and summative assessment in a general practice clinical attachment and its relationship to final medical school examination performance. Med Educ. 2001; 35(9): 841-6.

16. Triviño X, Vásquez A, Mena A, López A, Aldunate M, Varas M, Lillo R, Wright A. Aplicación del Examen Clínico Objetivo Estructurado (OSCE) en la evaluación final del internado de pediatría en dos Escuelas de Medicina. Rev Med Chil. 2002; 130(7): 817-824.

17. Mendel N, Fuks J, Levi T, Fernández M, De Preliasco VF, Amantea A. Examen clínico objetivo y estructurado (ECOE): una propuesta innovadora en la evaluación de la Odontopediatría. Revista de la Facultad de Odontología (UBA). 2005; 30(49): 31-36.

18. Demestre X. Evaluación de competencias en neonatología. Experiencias en Cataluña. Bol. S Vasco-Nav Pediatr. 2005; 38(1): 16-19.

19. Sibert L, Mairesse JP, Aulanier S, Olombel $\mathrm{P}$, Becret $\mathrm{F}$, Thiberville J et al. Introducing the objective structured clinical examination to a general practice residency programme: results of a French pilot study. Med Teach. 2001; 23(4): 383-388.

20. Hodges B, Regehr G, Hanson M, McNaughton N. Validation of an Objetive Structured Clinical Examination in Psychiatry. Academic Medicine. 1998; 73(8): 910-2.

21. Miranda T, Gasic M, Salas J, Mandiola E, Castillo M, Velasco C. Validez y confiabilidad de OSCE en Postgrado. Comunicaciones Panel. Educ Méd. 2003; 6(3): 53-63.

22. Bustos M, Chiolo M, Cutri A, Noguerol M, Ossorio M, Torres F et al. Examen clínico estructurado objetivo para residentes de pediatría. Presentación de una experiencia. Arch Argent Pediatr. 2007; 105 (4): 333-341.
23. Blanco MA, Oliva L, Bosch R, Menéndez L, Suárez R. Evaluación del razonamiento clínico. Educ Med Super [Internet] 2005 [citado 22 noviembre 2010]; 19(4). Disponible en http://scielo.sld.cu

24. Harden RM, Stevenson M, Downie WW, Wilson GM. Assessment of clinical competence using objective structured examination. Br Med J. 1975; 1(5955): 44751.

25. Harden RM. What is an OSCE? Med Teach. 1988; 10(1): 19-22.

26. Pantoja MA, Cabezas M, Illesca M, Navarro N, Sandoval P, San Martín S et al. Manual de Evaluación para Carreras de la Salud. Temuco: Universidad de La Frontera; 2002.

27. Crespo A, Maceiras R, Paredes I, Sixto M, Valiente D. Comparación de los resultados del examen objetivamente estructurado con el examen tradicional de morfología en Licenciatura en Enfermería 2002. Revista Avances [Internet] 2002 [citado 9 agosto 2010]; 4(1). Disponible en: http://www.ciget.pinar.cu/Revista/ No.2002-1/enfermeria.htm

28. Becerra R, Caballero E. Estrés frente a evaluación de competencias clínicas en base a Objective Structured Clinical Examination (OSCE). Revista Educación en Ciencias de la Salud. 2008; 5(2): 103-107.

29. Antolín R, Puialto MJ, Moure ML, Quinteiro T. Situaciones de las prácticas clínicas que provocan estrés en los estudiantes de enfermería. Enfermería Global. 2007; 6(10): 1-12.

30. Sandín MP. Investigación cualitativa en educación. Fundamentos y tradiciones. 1ra ed. Madrid: McGraw-Hill/Interamericana de España; 2003.

31. Programa Enfermería Básica del Niño, Adolescente y Adulto. Temuco: Universidad de La Frontera; 2007.

32. Glaser B, Strauss A. The discovery of Grounded Theory. Chicago: Aldine; 1967.

33. Miles MB, Huberman AM. Qualitative 
data analysis: an expanded sourcebook. Newbury Park, CA: Sage; 1994.

34. Van Maanen J. Qualitative Methodology. California: Sage; 1983.

35. Guba E, Lincoln Y. Naturalistic Inquiry. London: Sage; 1985; 37-40.

36. Lorda PS. El Consentimiento Informado: teoría y práctica. Med Clin. 1993; 101(5):
174-182.

37. Villalobos E, Guerrero M. Innovación en evaluación de competencias clínicas en el Pregrado de la Carrera de Obstetricia y Puericultura a través del método OSCE. Revista Colegio de Matronas. 2005; 13(2): 3-10. 